\title{
Van der Waals-Like Instability in Suspensions of Mutually Repelling Charged Colloids
}

\author{
René van Roij and Jean-Pierre Hansen \\ Laboratoire de Physique de l'Ecole Normale Supérieure de Lyon (URA 1325 du CNRS) \\ 46, Allée d'Italie, 69364 Lyon Cedex 07, France
}

(Received 19 June 1997)

\begin{abstract}
We show theoretically that the purely repulsive screened-Coulomb (or Derjaguin-Landau-VerweyOverbeek) interaction between charged colloidal particles is compatible with gas-liquid, gas-solid, and solid-solid coexistence in colloidal suspensions of low ionic strength of about $10^{-6} \mathrm{~mol} / \mathrm{liter}$. This finding may partially resolve the ongoing debate on attractions between like-charged particles. [S0031-9007(97)04326-3]
\end{abstract}

PACS numbers: 82.70.Dd, 64.10.+h, 83.20.Di

Colloidal particles with typical diameters in the range of $10-10^{3} \mathrm{~nm}$ carry, when suspended in a polar medium like water, a net negative charge of the order of $10^{2}-10^{4}$ electron charges. These macroionic charges are compensated by positively charged microscopic counterions carrying typically only one unit charge. In addition, suspensions usually contain added salt ions, typically in concentrations of $10^{-6}-10^{-2} \mathrm{~mol} / \mathrm{liter}$. In the high salt concentration regime, the phase behavior of such colloidal suspensions is well described by the Derjaguin-LandauVerwey-Overbeek (DLVO) theory [1]. An important result of this theory is the effective pair potential $v(r)$ between two identical macroions with total charge $-Z e$ and radius $R$, at center-to-center distance $r$. It is given by the screened-Coulomb form

$$
v(r)=\frac{Z^{2} e^{2}}{\epsilon}\left(\frac{\exp (\kappa R)}{1+\kappa R}\right)^{2} \frac{\exp (-\kappa r)}{r},
$$

where $-e$ is the electron charge, and $\epsilon$ the dielectric constant of the suspending medium. The bracketed factor accounts for the exclusion of microions from the interior of the colloids, while the range of $v(r)$ is controlled by the screening parameter $\kappa$, related to the average microion density $n$ and temperature $T$ by

$$
\kappa^{2}=\frac{4 \pi e^{2} n}{\epsilon k_{B} T},
$$

with $k_{B}$ the Boltzmann constant. Here, and henceforth, we assume for simplicity that all microions are monovalent. The DLVO potential of (1) is a cornerstone of colloid science, and accounts for many phenomena observed in suspensions of charged colloidal particles. For instance, the purely repulsive form of (1) explains the experimentally observed fluid-solid coexistence at sufficiently high colloid densities.

There are, however, other experimental results that do not seem to fit into the framework of the DLVO theory. These experiments are invariably performed at extremely low salt concentrations of the order of $10^{-6} \mathrm{~mol} / \mathrm{liter}$. For instance, the measured lattice spacings in crystalline phases of such quasideionized suspensions were found to be smaller than expected on the basis of a space-filling structure of the known colloid density [2]. A natural explanation for this would be that the dense crystalline phase is not pure, but coexists with a much more dilute gas phase. More evidence of phase coexistence has been provided by the observation of extremely dilute voids (a gas phase) in the bulk of an apparently homogeneous deionized suspension (a liquid) [3]. In fact, even a fully equilibrated gas-liquid coexistence has been reported, although this observation aroused some controversy [4].

According to standard liquid-state theory, the above mentioned coexistence of dilute and dense phases would require the existence of long-ranged attractions between the colloidal particles. Such attractions could be provided by the van der Waals forces between the colloids. However, the Hamaker constants that would qualitatively explain the observations are at least 1 order of magnitude too large. Hence attraction mechanisms of electrostatic origin have been proposed, at the cost, though, of sacrificing the well-established purely repulsive DLVO potential. In this Letter we argue that the observations at low salt concentrations can still be explained qualitatively within the framework of the DLVO potential (1).

The essence of our argument is most easily seen by neglecting, for the moment, the influence of added salt in the colloidal suspension. We thus consider a thermodynamic system of volume $V$ and dielectric constant $\epsilon$, suspending (i) $N_{m}=V n_{m}$ identical macroions of negative charge $-Z e$ and hard-sphere diameter $D=2 R$, and (ii) $N_{c}=$ $Z N_{m}=V n_{c}$ positive pointlike counterions. The Hamiltonian $H=H_{m}+H_{c}+V_{m c}$ of this system consists of a sum of the Hamiltonians $H_{m}$ and $H_{c}$ of the macro- and counterions, respectively, and of the macroion-counterion interaction term $V_{m c}$. For later reference we write the macroion Hamiltonian explicitly as

$$
H_{m}=K_{m}+\frac{1}{2} \sum_{i \neq j=1}^{N_{m}}\left(v_{\mathrm{hs}}\left(R_{i j}\right)+\frac{Z^{2} e^{2}}{\epsilon} \frac{1}{R_{i j}}\right),
$$

with $K_{m}$ the kinetic energy, $v_{\mathrm{hs}}\left(R_{i j}\right)$ the hard-sphere pair potential between two spheres of radius $R$ at positions $\mathbf{R}_{i, j}$, and $R_{i j}=\left|\mathbf{R}_{i}-\mathbf{R}_{j}\right|$. At fixed inverse temperature $\beta=1 / k_{B} T$, the total Helmholtz free energy $F$ of 
this system can formally be written as $\exp [-\beta F]=$ $\operatorname{Tr}_{m} \exp \left[-\beta H_{m}^{\text {eff }}\right]$, with the effective macroion Hamiltonian $H_{m}^{\text {eff }}=H_{m}+F^{\prime}$. Here $F^{\prime}$ is given by $\exp \left[-\beta F^{\prime}\right]=$ $\operatorname{Tr}_{c} \exp \left[-\beta\left(H_{c}+V_{m c}\right)\right]$, and the trace $\operatorname{Tr}_{m(c)}$ is short for the phase-space integral over the macro(counter)ion degrees of freedom $[5,6]$. Clearly, $F^{\prime}$ can be interpreted as the Helmholtz free energy of an inhomogeneous fluid of counterions in the external field of macroions at fixed positions $\mathbf{R}_{j}$. Here we calculate $F^{\prime}$ using the framework of density functional theory $[7,8]$, which is based on the existence of a functional $\mathcal{F}\left[\rho^{(1)}\right]$ of possible counterion density profiles $\rho^{(1)}(\mathbf{r})$. The thermodynamic equilibrium profile $\rho(\mathbf{r})$ is that profile that minimizes $\mathcal{F}$ while satisfying the normalization condition $\int d \mathbf{r} \rho(\mathbf{r})=N_{c}$, and $F^{\prime}=\mathcal{F}[\rho]$.

Unfortunately, the exact functional form of $\mathcal{F}$ is unknown, and hence approximations are unavoidable. Here we focus on a simple quadratic mean-field functional, in which (i) the logarithmic ideal-gas term is expanded to quadratic order about the homogeneous distribution, and (ii) the counterion-counterion correlations are neglected. This functional reads

$$
\begin{aligned}
\mathcal{F}\left[\rho^{(1)}\right]= & F_{i d}\left(N_{c}, V, T\right)+\frac{k_{B} T}{2 n_{c}} \int d \mathbf{r}\left[\rho^{(1)}(\mathbf{r})-n_{c}\right]^{2} \\
& +\int d \mathbf{r} \rho^{(1)}(\mathbf{r}) U(\mathbf{r}) \\
& +\frac{e^{2}}{2 \epsilon} \int d \mathbf{r} d \mathbf{r}^{\prime} \frac{\rho^{(1)}(\mathbf{r}) \rho^{(1)}\left(\mathbf{r}^{\prime}\right)}{\left|\mathbf{r}-\mathbf{r}^{\prime}\right|}
\end{aligned}
$$

where $F_{i d}\left(N_{c}, V, T\right)=N_{c} k_{B} T\left[\ln \left(n_{c} \Lambda^{3}\right)-1\right]$ is the homogeneous ideal gas term with $\Lambda$ the thermal wavelength. The second term of (4) represents the lowest order inhomogeneous ideal gas contribution, the third one the coupling to the macroionic external field $U(\mathbf{r})$, and the fourth one the purely Coulombic counterion-counterion interaction. We assume that $U(\mathbf{r})=\sum_{j=1}^{N_{m}} u\left(\mathbf{r}-\mathbf{R}_{j}\right)$ is a multicentered sum of a macroion-microion pair interaction

$$
u(\mathbf{r})=\left\{\begin{array}{lll}
-\frac{Z e^{2}}{\epsilon} \frac{1}{|\mathbf{r}|} & |\mathbf{r}|>R, \\
-\frac{Z e^{2}}{\epsilon} \frac{1-\alpha}{R} & |\mathbf{r}|<R,
\end{array}\right.
$$

which is purely Coulombic outside the macroionic core and constant, parametrized by $\alpha$, within. In principle, one should consider the hard-core limit $\alpha \rightarrow \infty$, but this is not compatible with the expansion of the full ideal-gas contribution in (4), which requires smooth density profiles. We will show below, however, that $\alpha$ can be chosen such as to mimic the hard core in the functional (4).

Because of the expansion of the ideal-gas term, the stationarity condition that follows from (4) is linear in $\rho(\mathbf{r})$, and can be solved easily. It yields $\rho(\mathbf{r})=\sum_{j=1}^{N_{m}} \rho_{0}(\mathbf{r}-$ $\mathbf{R}_{j}$ ), where the "orbitals" $\rho_{0}(\mathbf{r})$ are given by

$$
\rho_{0}(\mathbf{r})= \begin{cases}\frac{\kappa^{2} Z_{>}}{4 \pi} \frac{\exp (-\kappa r)}{r} & r>R, \\ \frac{\kappa^{2} Z_{<}}{4 \pi} \frac{\sinh (\kappa r)}{r} & r<R,\end{cases}
$$

with the effective charges

$$
\begin{aligned}
& Z_{>}=Z\left((1-\alpha) \frac{\sinh (\kappa R)}{\kappa R}+\alpha \cosh (\kappa R)\right), \\
& Z_{<}=Z\left(\frac{\exp (-\kappa R)}{\kappa R}\right)[1-\alpha(\kappa R+1)] .
\end{aligned}
$$

The density profile (6) can be inserted back into the functional (4) to yield $F^{\prime}=\mathcal{F}[\rho]$, which leads to

$$
\begin{aligned}
H_{m}^{\mathrm{eff}}= & K_{m}+\frac{1}{2} \sum_{i \neq j}^{N_{m}}\left(v_{\mathrm{hs}}\left(R_{i j}\right)+\frac{Z_{>}^{2} e^{2}}{\epsilon} \frac{\exp \left(-\kappa R_{i j}\right)}{R_{i j}}\right) \\
& +F_{i d}\left(N_{c}, V, T\right)-\frac{Z^{2} e^{2}}{2 \epsilon} \kappa N_{m} h(\alpha ; \kappa R)
\end{aligned}
$$

where

$$
\begin{aligned}
h(\alpha ; x)= & (1-\alpha)^{2} \frac{\exp (-2 x)-1+2 x}{2 x^{2}} \\
& +2 \alpha(1-\alpha) \frac{1-\exp (-2 x)}{2 x} \\
& +\alpha^{2} \frac{\exp (-2 x)+1}{2} .
\end{aligned}
$$

Here the screening parameter $\kappa$ is given by (2) with $n=n_{c}$. A comparison of the first line of (8) with the bare Hamiltonian (3) shows the well-known result that integrating out the counterions yields screened-Coulomb (or Yukawa) instead of bare-Coulomb repulsions between the macroions. The second line of (8) does not depend on the coordinates $\mathbf{R}_{j}$ of the macroions, and would naively be disregarded as an irrelevant constant energy offset. However, the key point of this Letter is that this offset depends nontrivially on the density of macroions, and can play an essential role in the total Helmholtz free energy $F$ of the system. The essence of the character of the final term of (8) is most easily understood for point macroions, as it follows from $h(\alpha ; \kappa R=0)=1$ that this term then equals $-Z^{2} e^{2} \kappa / 2 \epsilon$ per macroion. This is of the order of the electrostatic energy of a macroion with "its own" counterion orbital of range $1 / \kappa$. As $\kappa \propto n_{m}^{1 / 2}$, the final term of (8) scales as $-V n_{m}^{3 / 2}$, and hence tends to drive a van der Waals loop in the equation of state. A term of this form also appears in the Debye-Hückel free energy of symmetric electrolytes, where it is now known to drive gas-liquid coexistence at sufficiently low temperatures [9]. Moreover, such a structure-independent term also has a well-known analog in the theory of liquid metals, where it is called the volume term $[5,6]$. The formal analogy between liquid metals and colloidal suspensions has been pointed out in Ref. [6], but the implications for the phase behavior of colloidal suspensions has, to the best of our knowledge, never been considered in detail. In this Letter we show that the second line of (8) can drive a spinodal instability for finite values of $R$, and lead to solid-solid, solid-gas, or liquid-gas coexistence. It is essential to realize that these phase coexistences occur despite the fact that the pairwise macroion-macroion interaction is purely repulsive. 
We have not yet included the effect of added salt, nor specified the parameter $\alpha$. The main complication of added salt is the presence of more than one microion density profile. It is straightforward, though, to generalize the functional (4), and to solve for the profiles separately. The first difference between the resulting effective Hamiltonian and that of (8) involves the definition of $\kappa$, which is still given by (2) but now with $n=Z n_{m}+2 n_{s}$, where $n_{s}$ is the number density of pairs of monovalent added salt ions. The second difference is the presence of homogeneous ideal gas terms for each microion species. Concerning the choice of the parameter $\alpha$, we first remark that the choice $\alpha=0$ gives $Z_{>}=Z \sinh (\kappa R) / \kappa R$ and $h(0 ; x)=$ $[\exp (-2 x)-1+2 x] / 2 x^{2}$, which leads to identical expressions as obtained by Sogami and Ise [10], who integrated the linearized Poisson-Boltzmann equation. Note, however, that their approach does not yield the ideal-gas term that is present in (8), and that they proceeded by calculating the pairwise Gibbs free energy, the interpretation of which is controversial [11]. Moreover, we see from (6) that the choice $\alpha=0$ leads to a nonvanishing microion density within the core about which it is centered. We argue, therefore, that the best choice to represent the hard core is $\alpha=$ $(1+\kappa R)^{-1}$, since then $\rho_{0}(r)=0$ for $r<R$. This choice, which will be used henceforth, leads to the effective charge $Z_{>}=Z \exp (\kappa R) /(1+\kappa R)$, and thus exactly reproduces the DLVO potential in (1). Using $h[1 /(1+x)$; $x]=1 /(1+x)$, we thus obtain the effective Hamiltonian

$$
\begin{aligned}
H_{m}^{\mathrm{eff}}= & K_{m}+\frac{1}{2} \sum_{i \neq j=1}^{N_{m}}\left[v_{\mathrm{hs}}\left(R_{i j}\right)+v\left(R_{i j}\right)\right] \\
& +F_{i d}\left(N_{s}, V, T\right)+F_{i d}\left(Z N_{m}+N_{s}, V, T\right) \\
& -\frac{Z^{2} e^{2}}{2 \epsilon} \frac{N_{m} \kappa}{1+\kappa R},
\end{aligned}
$$

with $v(r)$ defined in (1) and $N_{s}=V n_{s}$. We assumed here that the released counterions are of the same species as the positive added salt ions. The first two terms of (10) constitute the Hamiltonian of a fluid of particles interacting with the pairwise DLVO potential. The final three terms are constants as a function of the macroion coordinates, but not as a function of the macroion density. In fact, one can easily see that these three terms are constant as a function of $n_{m}$ only in the high salt concentration regime $n_{s} \gg Z n_{m}$, while the $n_{m}$ dependence is very strong if $n_{s} \leq Z n_{m}$. In the latter case, the final term of (10) can drive a spinodal instability. On the basis of this, one can understand qualitatively why theories based only on the first two terms of (10) cannot explain the observed phase behavior at low salt concentrations, while they do describe the physics at higher salt concentrations qualitatively correctly. In the sequel we show that the cross over from the low to the high salt concentration regime takes place at $n_{s} \sim 10^{-6} \mathrm{~mol} /$ liter for typical values for $R$ and $Z$, which is in the regime of the experimentally observed two-phase structures.
To deduce the phase diagram that follows from the effective Hamiltonian (10), one first has to determine the Helmholtz free energy $F$. This is done here variationally using the Gibbs-Bogolyubov (GB) inequality, where the reference fluid phase is the hard-sphere fluid, and the reference solid phase the Einstein crystal [12]. Once $F$ is known, the phase coexistence is determined by imposing the standard conditions of equal osmotic pressure and chemical potential (both for the macroions and added salt), e.g., via suitable common tangent constructions. A drawback of the use of the GB inequality is that it underestimates the stability of the body-centered cubic phase (BCC) with respect to that of the face-centered cubic phase (FCC) and the fluid phase (F). It follows from our calculations that the F-BCC coexistence is always preempted by F-FCC, whereas it is known that $\mathrm{BCC}$ can be stable at very low ionic strength. However, this drawback, which was also encountered in Ref. [12], does not affect our main conclusions, since the free energy difference between FCC and BCC is much smaller than that between F and either FCC or BCC; we can simply not distinguish FCC and BCC accurately.

Below we present three phase diagrams that follow from the effective Hamiltonian (10) and the procedure described above. We consider aqueous colloidal suspension at room temperature, and thus set $e^{2} / \epsilon k_{B} T=$ $7.2 \AA$. We also take into account the dissociation of water; the $\mathrm{H}^{+}$and $\mathrm{OH}^{-}$ions each provide an offset of $n_{0}=$ $1 \times 10^{-7} \mathrm{~mol} /$ liter to the total ion density in the fluid. In the actual calculations we thus define $\kappa$ as in (2), but with $n=Z n_{m}+2 n_{s}+2 n_{0}$. Of course, this offset is negligible at high salt concentrations or high colloidal density, but is relevant at lower total ionic strength. The three diagrams, presented in the $\eta-n_{s}$ plane with the colloidal packing fraction $\eta=(4 \pi / 3) R^{3} n_{m}$, all correspond to the same realistic macroionic surface-charge density $-Z e / 4 \pi R^{2}=-0.546 e / \AA^{2}$, but differ in diameter $D$ and hence in total charge number $Z$. The following picture emerges. If $Z$ is small enough (this case is not shown in a figure) there is no spinodal instability, and the only stable coexistence is F-FCC. The mechanism of this transition is essentially that of the hard-sphere freezing transition, which leads to coexisting phases of almost the same densities (narrow coexistence). At $Z=$ 6881 and $D=633 \mathrm{~nm}$ [Fig. 1(a)] the total charge is sufficiently large to cause an instability that drives a demixing transition in the FCC phase at $n_{s}<2 \mu \mathrm{mol} /$ liter. The salt concentration in the dense phase is somewhat lower than in the coexisting dilute phase. The main difference with ordinary phase separation is the existence of an upper and a lower critical point, and a reentrant phenomenon. Increasing $Z$ by only $3 \%$, so that $Z=7300$ and $D=$ $652 \mathrm{~nm}$ [Fig. 1(b)], leads to a region of instability that intersects the narrow F-FCC coexistence. Because of the reentrant phenomenon, this leads to two F-FCC-FCC triple points, and to two distinct FCC-FCC coexistence regions separated by a broad F-FCC coexistence. In this case, 

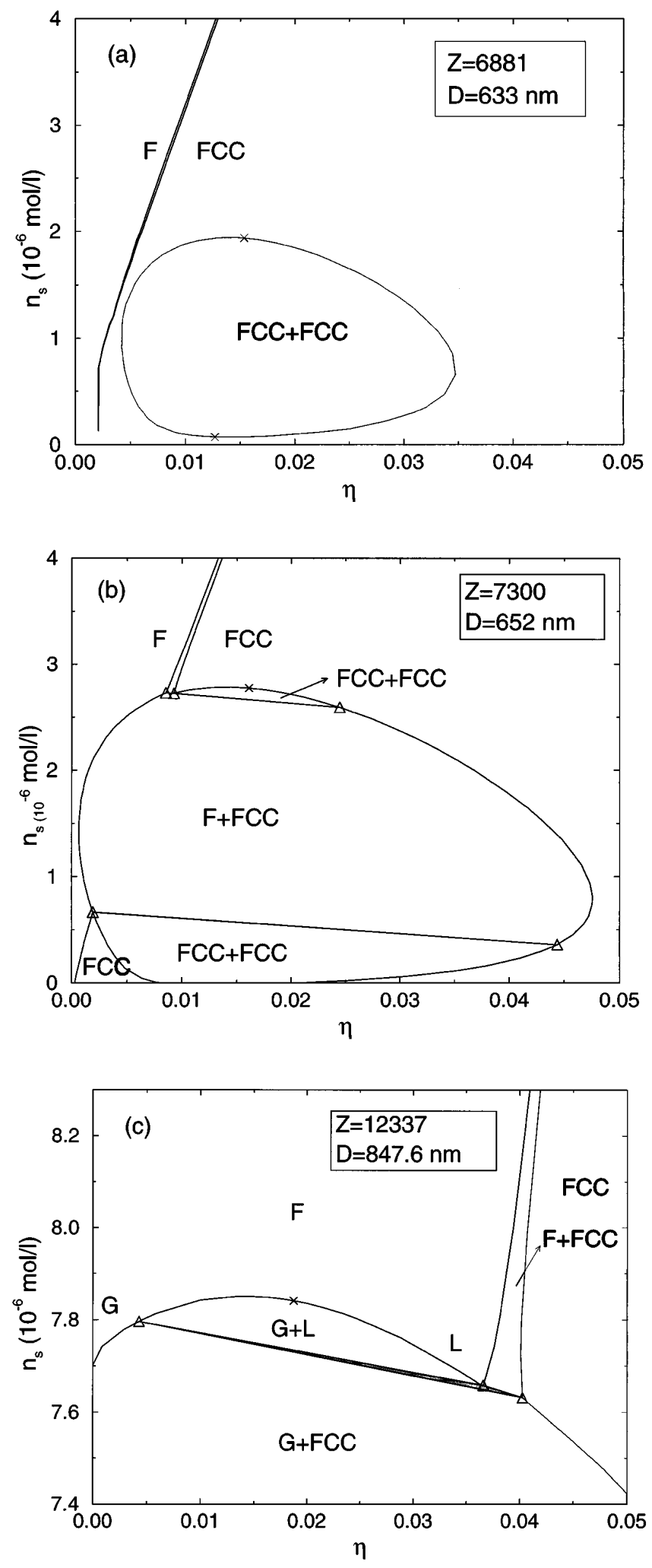

FIG. 1. Room temperature phase diagrams of aqueous monodisperse suspensions of colloidal particles of diameter $D$ and charge number $Z$, as a function of the colloidal packing fraction $\eta$ and the concentration $n_{s}$ of monovalent added salt ion pairs. The phases are fluid (F), FCC-solid (FCC), liquid $(\mathrm{L})$, and gas $(\mathrm{G})$. The critical points are indicated by $\times$, the triple points by $\Delta$. Note that the F-FCC coexistence regime becomes extremely narrow in (a) and (b) at $n_{s} \lesssim 1 \mu \mathrm{mol} / \mathrm{liter}$. the lower critical point does no longer exist. Note that the present values of $Z$ and $D$ are precisely those used in recent experiments by Larsen and Grier [13], who showed the existence of long-lived metastable crystallites at $\eta \approx 0.02$ and $n_{s} \leq 2.5 \mu \mathrm{mol} /$ liter. Although their nonequilibrium experiments can never be fully explained by the present equilibrium theory, it is tempting to argue that their long-lived metastable crystallites are not separated from the equilibrium fluid phase by a narrow hard sphere like fluid-solid coexistence, but instead by a very broad one as in Fig. 1(b) at $n_{s} \leq 2.5 \mu \mathrm{mol} /$ liter. Increasing $Z$ again by some $30 \%$, so that $Z=12337$ and $D=847.6 \mathrm{~nm}$ [Fig. 1(c)], leads to a shift of the critical point into the fluid phase, and hence to gas-liquid (GL) and G-FCC coexistence. Note, however, the different scale of Fig. 1(c).

The theory we have presented here shows the possibility of a spinodal instability - and hence coexistence between dilute and dense phases - without the presence of effective attractions between the colloidal particles. Although this does not mean that such attractions never exist, we argue that their existence cannot be inferred on the basis of observations of two-phase coexistence in experiments, as is often done $[3,14]$. The source of the instability is the density dependence of the electrostatic energy of each macroion with its "own" cloud of microions.

It is a pleasure to thank Thierry Biben and Emmanuel Trizac for numerous discussions, and Marjolein Dijkstra for a critical reading of the manuscript. This work was made possible by financial support from the "Région Rhône-Alpes."

[1] E. J. W. Verwey and J. Th. G. Overbeek, Theory of the Stability of Lyotropic Colloids (Elsevier, Amsterdam, 1948).

[2] N. Ise and M. V. Smalley, Phys. Rev. B 50, 16722 (1994).

[3] K. Ito, H. Hiroshi, and N. Ise, Science 263, 66 (1994); B. V.R. Tata, E. Yamahara, P. V. Rajamani, and N. Ise, Phys. Rev. Lett. 78, 2660 (1997).

[4] B. V.R. Tata, M. Rajalakshmi, and A. K. Arora, Phys. Rev. Lett. 69, 3778 (1992); T. Palberg and M. Würth, Phys. Rev. Lett. 72, 786 (1994).

[5] R. Evans and M. Hasegawa, J. Phys. C 14, 5225 (1981).

[6] M. J. Grimson and M. Silbert, Mol. Phys. 74, 397 (1991).

[7] R. Evans, Adv. Phys. 28, 143 (1979).

[8] H. Löwen, J. P. Hansen, and P. A. Madden, J. Chem. Phys. 98, 3275 (1993).

[9] M.E. Fisher and Y. Levin, Phys. Rev. Lett. 71, 3826 (1993), and references therein.

[10] I. Sogami and N. Ise, J. Chem. Phys. 81, 6329 (1984).

[11] C. E. Woodward, J. Chem. Phys. 89, 5140 (1988).

[12] W.H. Shih and D. Stroud, J. Chem. Phys. 79, 6254 (1983); W. Y. Shih, I. A. Aksay, and R. Kikuchi, J. Chem. Phys. 86, 5127 (1987).

[13] A.E. Larsen and D. G. Grier, Nature (London) 385, 230 (1997).

[14] N. Ise and H. Yoshida, Acc. Chem. Res. 29, 3 (1996). 